\title{
A Educação Ambiental e a utilização de Oficinas Pedagógicas na formação da Cidadania.
}

Environmental education and the use of Pedagogical Workshops in the formation of

Citizenship.

\author{
Rita de Cássia de Souza Zara25 \\ Bartolomeu Tavares ${ }^{26}$
}

Artigo recebido em para publicação em set/2013 e aceito para publicação em mar/2014

\section{RESUMO}

Esse projeto apresenta uma abordagem a respeito da relação entre educação ambiental e sua importância na formação de uma postura critica cidadã do aluno, a partir de aulas com oficinas. Empreende inicialmente um levantamento preliminar sobre a consideração prévia dos alunos de um curso técnico de Cascavel, a respeito da temática educação ambiental, e, após a aplicação das oficinas pedagógicas, aplicou-se uma coleta de dados para observar o conhecimento adquirido pelos alunos. Nesse sentido, constatou-se que a modificação da dinâmica das aulas propiciou uma participação mais ativa e maior envolvimento dos alunos com o processo de aprendizagem de forma a possibilitar uma concretização prática desse conhecimento obtido, no cotidiano do aluno, de maneira que o mesmo atue como um multiplicador ambiental, junto a sua comunidade e exerça uma postura crítica cidadã perante os problemas ambientais.

Palavras-chave: Educação ambiental. Formação de multiplicadores. Oficinas.

\section{ABSTRACT}

This project presents an approach according to the relationship between the environmental education and its importance on the students' background, who can be citizens and critic, through classes with workshops. It, initially, undertakes a preliminary survey concerning the students' considerations of a technical course in Cascavel, at environmental education and after the workshops implementation, a pedagogical data collection was applied to observe the students' acquired knowledge. Thus, it was found out that the change on dynamics of classes provided a more active participation and involvement of students in the learning process so that it could supply a concrete gained knowledge in the student's daily life, so that he can act out as a multiplier regarding the environment, in his neighborhood and perform as a citizen with critical attitudes towards the environmental problems.

Keywords: Workshops. Environmental education. Training of multipliers.

25 Graduada em Tecnologia de Alimentos pela Universidade Tecnológica Federal do Paraná UTFPR-Medianeira, Graduada em Ciências Biológicas e Especialista em Gestão Ambiental e de Recursos Hídricos pela Universidade Estadual do Oeste do Paraná - UNIOESTE. Técnica de laboratório na Companhia de Saneamento do Paraná - SANEPAR. E-mail: rcsouza@sanepar.com.br ${ }^{26}$ Graduado em Ciências Biológica pela Universidade Federal de Juiz de Fora - UFJF, Mestrado em Botânica pela Universidade Federal do Paraná - UFPr e Doutorado em Biologia Vegetal pela Universidade Estadual Paulista/Rio Claro - UNESP. Professor efetivo na Universidade Estadual do Oeste do Paraná - UNIOESTE. Email: bartolomeu.tavares@unioeste.br e bartolomeutavares@hotmail.com 


\section{INTRODUÇÃO}

Fazer com que alunos reflitam sobre suas práticas cotidianas e as sistematizem através da escrita, no processo de aprendizagem, não é um exercício simples.

Nesta perspectiva, "o trabalho escolar voltado à implementação de propostas metodológicas interdisciplinares exige a conjunção de uma série de fatores, a começar pelo reconhecimento de que a questão ambiental perpassa várias áreas do conhecimento" (SEGURA, 2001, p.136).

Acreditamos que as atividades práticas e de oficinas devam ser diversificadas para estimular o desenvolvimento dos alunos.

Segundo Zóboli (1994), a aprendizagem não se dá pelo fato de ouvir e folhear o caderno, mas a partir de uma relação teórico-prática, com intuito não de comparar, mas de despertar interesse nos alunos, a fim de gerarem-se discussões e melhor aproveitamento das aulas.

Um dos erros das aulas tradicionais de Biologia é que o professor se interpõe entre a natureza e o aluno, servindo de intérprete, transmitindo informações, tendo como recurso exclusivo o livro didático e sua transcrição no quadro (FROTA, 1982). No entanto, não é suficiente "usar o laboratório" ou "fazer experiência", as atividades experimentais não devem ser usadas somente para provar aos alunos leis e teorias, pois são pobres aos objetivos de formação e apreensão de conhecimentos.

Apesar da importância das aulas práticas com oficinas pedagógicas, as mesmas não são amplamente utilizadas, devido à falta de tempo para preparação do material, insegurança dos professores para controlar a classe, disponibilidade de materiais, estrutura e conhecimento para organizar experiências.

Muitas vezes o aluno possui um conceito sobre determinado assunto e isso pode interferir na prática, pois esse passa a basear-se em seu próprio conhecimento. O educador deve tomar o cuidado de levantar as concepções já formadas e moldá-las até chegar realmente em sua meta final (FROTA, 1982).

Ainda segundo Silvestre (2001), a utilização desses recursos propicia aos alunos a verificação de diversos fenômenos e processos naturais que estão no ambiente e possibilita explorar aspectos relacionados com impactos provocados pela ação humana nos ambientes e sua interação com o trabalho produtivo e projetos sociais. 
Com este trabalho, objetivou-se investigar o nível de conhecimento dos alunos, a respeito das temáticas abordadas em aula, em específico sobre sua postura crítico-participativa na interação do aluno com o ecossistema, de forma que, a partir do conhecimento do perfil de cada aluno, possam ser desenhadas estratégias de ensino, em forma - por exemplo - de oficinas para que tais conhecimentos prévios possam ser modificados visando a uma participação cidadã.

\section{METODOLOGIA}

O projeto, com início em março de 2008, teve como proposta de abordagem científica o fazer educação ambiental, a partir das práticas vivenciadas na comunidade e no espaço escolar, sendo as práticas partilhadas e refletidas de forma sistemática no processo de construção do conhecimento, a partir de diversas disciplinas do currículo, visando à importância da investigação científica na pesquisa de ciências para o despertar do aluno.

Para verificar as pré-concepções iniciais dos alunos, quanto ao tema trabalhado, e as pós-concepções adquiridas no final do desenvolvimento do projeto, foram elaborados questionários, ambos aplicados tanto no início quanto no final do trabalho, submetidos e aprovados no Comitê de Ética da Unioeste.

Durante o desenvolver do trabalho, utilizou-se de aulas expositivas dialogadas problematizadoras, com auxílio de material audiovisual - multimídia, para abordar o conteúdo teórico. Os alunos foram investigados sobre seus conhecimentos, durante as aulas. Na sequência, os alunos foram separados em equipes para a realização das atividades de oficina. Após as oficinas e visitas técnicas, os alunos passaram por uma avaliação, cobrada, na forma de relatórios de atividades práticas, para possibilitar a observação do conhecimento adquirido por eles.

Nesta expectativa, as oficinas surgiram como um projeto-piloto. Durante o processo de inclusão do aluno à iniciação científica, trabalhos coletivos foram realizados, tendo como subsídios estudos científicos sobre a importância do saneamento na manutenção do equilíbrio do ecossistema do planeta e seu contexto com o cotidiano e da comunidade escolar, e sobre a problemática do lixo urbano, assoreamento de lagos e contaminação de solos, que são fatos ocorridos e verificados no município. 
Os alunos inclusos neste estudo são da rede pública do município de Cascavel, matriculados no Centro Estadual Educacional Profissionalizante - CEEP. Foi trabalhado com alunos tanto do sexo feminino como masculino e a faixa etária variou entre 14 e 23 anos. Para os participantes com idade inferior a 16 anos, foi aplicado questionário, mediante a autorização do responsável.

Como instrumento da coleta de dados, foram aplicados dois questionários conjuntamente, antes e depois do desenvolvimento do projeto. Os dados qualitativos foram obtidos a partir das respostas às perguntas após a realização das oficinas e atividades de campo. Observou-se também o comportamento e os comentários dos alunos durante toda a pesquisa, como forma de interagir sobre suas percepções.

Os dados obtidos a partir dos questionários foram tabulados, expressos em valores percentuais e analisados graficamente pelo programa Excel Microsoft $\Theta$. Os resultados gráficos estão apresentados em sua forma percentual.

\section{RESULTADOS E DISCUSSÃO}

Para análise do perfil socioambiental dos alunos, as perguntas apresentadas nos questionários pré e pós-testes ficaram divididas em dois focos principais: o interesse dos alunos em participar de atividades de pesquisa e oficinas e a percepção dos impactos ambientais em seu cotidiano.

Atividades práticas de oficinas permitem, e até mesmo estimulam, a participação ativa dos alunos na construção do processo dinâmico da aprendizagem, portanto, contribuem para que se possa vivenciar e aplicar concretamente em suas ações o conhecimento obtido e possibilitar, assim, uma melhoria em sua qualidade de vida, de forma que o aluno possa se tornar um multiplicador de conhecimento, junto a sua comunidade exercendo uma postura crítica-cidadã (MUCELIN, 2003).

A educação socioambiental não é a solução para o Planeta, é solução para as pessoas que vivem em sociedades (CORREA, 2000). A ligação de temas ambientais com temas sociais, e vice-versa, resulta em um facilitador do efetivo exercício da cidadania, o qual geraria uma rede de relações entre pessoas e grupos com interesses socioambientais comuns, destacando-se a importância da preservação e conservação do meio ambiente, para a melhoria da qualidade de vida de todos (DANTAS, 1998). 
$\mathrm{Na}$ aplicação da pesquisa em relação à percepção dos alunos em torno dos impactos ambientais, pré-teste, $43 \%$ dos alunos citam fontes de poluição no rio e em suas proximidades, com destaque para a existência de despejos clandestinos de esgoto. E 19\% observaram fontes e processos degradativos das condições naturais, sendo destacada a presença de lixo urbano. O restante dos alunos não soube identificar as causas de poluição. Comparando-se as respostas obtidas na pesquisa pré e pós-testes, pôde-se perceber uma elevação em relação à percepção ambiental dos alunos, para as fontes de poluição no rio, presente no bairro. Isso ressalta a importância das aulas vivenciais e os diagnósticos em campo, desenvolvidos durante a realização do projeto, como ferramenta para a conscientização ambiental dos alunos.

Quando perguntado aos alunos, na abordagem pré-teste, sobre a presença de Mata Ciliar para proteção do rio presente no bairro, 39\% dos alunos citaram a ausência de cobertura vegetal. E no pós-teste, pela simples observação da ação do homem sobre o ecossistema, $83,3 \%$ dos alunos relataram não que conheciam as características naturais da região.

Durante as atividades de construção de ambientes com impactos ambientais, muitos alunos declararam a importância tanto da conservação do meio ambiente quanto da relação entre fauna e flora para a manutenção do equilíbrio do ecossistema. E cuidaram com muita dedicação e carinho dos minhocários construídos por eles, inclusive alguns alunos tiveram interesse em adotar o minhocário em suas casas, comparando-o a seu "bichinho de estimação".

A observação dos dados pré e pós-teste mostra que o rio do bairro e suas intermediações são frequentados por crianças que os utilizam como área de lazer. Tal diagnóstico indica ser necessária uma tomada de solução por parte das autoridades municipais responsáveis, tanto no investimento em políticas públicas quanto para melhor estrutura do local e melhoria da conscientização ambiental dos moradores, através da melhoria na educação.

Não foi verificada a preocupação ou ação efetiva das autoridades responsáveis por $62 \%$ dos alunos na avaliação pré-teste e por $83,3 \%$ dos alunos na pesquisa pós-teste.

Tal resultado reforça a importância de investimentos por parte da autoridade municipal responsável, em infraestrutura e educação para assegurar que a 
população possa ter condições dignas de habitação e moradia e exerça sua cidadania.

Os resultados abaixo objetivaram a verificação dos conhecimentos prévios dos alunos, assim como o ganho obtido, após a realização das oficinas pedagógicas, para a abordagem da temática ambiental de maneira a contribuir com a formação de multiplicadores ambientais. Para a observação da percepção final dos alunos, após o desenvolvimento do trabalho, foram também consideradas as respostas discursivas obtidas pelos relatórios.

Quando perguntado aos alunos, na pesquisa pré-teste, com quais disciplinas do curso técnico em Meio Ambiente, eles se identificavam mais, pôde-se perceber que $66 \%$ dos alunos aderem sua preferência à disciplina de Ciências e Biologia e apenas 8\% mostram seu interesse pela Matemática. Na pesquisa pós-teste, percebeu-se um aumento para a disciplina de Matemática, porém prevaleceu o interesse pela Ciência e Biologia. Com base nos dados, pode-se avaliar a importância de atividades experimentais de caráter multidisciplinar para abordagem da temática educação ambiental, ou seja, para que o aluno compreenda o processo envolvido na determinação das propriedades físico-químicas de amostras de água e esgoto, como foi trabalhado em aulas práticas, faz-se necessária a interligação de algumas disciplinas, normalmente vistas isoladamente.

Para Brasil (1998), como a Educação Ambiental é um tema transversal que vem permeando os conteúdos curriculares das disciplinas, os Parâmetros Curriculares Nacionais - PCN - incluíram esse tema nos currículos de ensino fundamental como uma forma de encontrar o caminho para se chegar à mudança de hábitos que levem à diminuição da degradação ambiental, promovam a melhoria da qualidade de vida e reduzam a pressão sobre os recursos naturais.

Ainda proposto pelos PCNs, como objetivo do ensino fundamental, o aluno deve ser capaz de questionar a realidade ao formular problemas e tratar de resolvêlos, utilizando para isso o pensamento lógico, a criatividade, a intuição, a capacidade de análise crítica, ao selecionar o procedimento e verificar sua adequação (BRASIL, 1998, p. 8).

Segundo Brasil (1998), os PCN não apresentam soluções prontas, mas colocam em debate as atividades escolares e a questão curricular. Constituem um material de referência atualizado sobre a função da escola, a importância dos 
conteúdos e temas transversais, e o tratamento que se Ihes deve ser dado. Tal informação vem a ressaltar a importância do desenvolvimento de projetos com metodologias práticas, por exemplo, as oficinas experimentais na abordagem temática ambiental nas escolas e para contribuir na formação crítica-cidadã do aluno.

Durante a apresentação dos resultados obtidos pelos relatórios de atividade prática, dividiram-se os dados observados em função dos módulos trabalhados; os quais seguem abaixo:

No módulo I, durante a simulação no laboratório das diferentes fases do tratamento da água, pelos alunos, foram observados os seguintes comentários:

\footnotetext{
"Se as pessoas não poluíssem tanto os rios, não precisaria usar tanto produto químico para tratar a água e a conta talvez diminuísse";

"Lá em casa nós tomamos água do poço e nem sabemos se ela é boa para nossa saúde";

"Legal, não imaginava, que além do cloro para eliminar as bactérias também se coloca flúor na água para evitar cárie".
}

No módulo II, Quanto às questões do relatório sobre a oficina de monitoramento da germinação das sementes. Na primeira pergunta sobre a relação entre os tipos de raízes desenvolvidas com o tipo de solo utilizado, foram obtidas as seguintes respostas:

\footnotetext{
"As raízes têm formas diferentes e vão se adaptar em solos diferentes, alguns solos são mais ricos em alimentos que outros";

"Em solos de argila, como os da nossa região, as plantas vão ter mais água, pois a argila segura mais água que a areia".
}

$\mathrm{Na}$ segunda pergunta do relatório, sobre a importância dos diferentes sistemas radiculares das plantas na conservação e manejo do solo e preservação dos rios, foram obtidas as seguintes respostas:

\footnotetext{
"As raízes são importantes para a nutrição e sustentação do solo e as plantas variam de solo para solo";

"As raízes permitem que bactérias importantes para o solo vivam sob sua superfície e as bactérias nutrem as plantas e o solo";

"As raízes são diferentes, e as raízes pivotantes, podem buscar no fundo do solo seus alimentos, enquanto as outras se alimentam na parte mais superior. As raízes firmam o solo e evitam que a chuva o leve para dentro dos rios e água fica mais limpa".
}

No módulo III, durante a abordagem sobre o gerenciamento do lixo urbano e a importância do saneamento para a manutenção da qualidade da vida, as quais evitam a veiculação de parasitoses ao homem, os alunos mostraram-se muito 
entusiasmados com a atividade "jogo didático sobre parasitoses". Todos relataram já terem presenciado casos com verminoses e que não gostariam que fossem com eles.

O intuito dessa atividade foi alcançado a partir do momento em que os alunos perceberam diretamente no ambiente, e também pelos diagnósticos de impactos ambientais feitos "in loco" durante as visitas técnicas realizadas. Todas essas informações enriquecessem as observações realizadas durante o jogo.

Junto aos materiais fornecidos pelas aulas teóricas, os alunos identificaram as espécies de parasitas e seus ciclos de vida e sistemas adaptativos e formas de prevenção da doença, assim como observaram alguns problemas enfrentados pelo falta de saneamento, ainda presentes em nosso município, como: lixo, poluição e esgoto. Atividades lúdicas pedagógicas são vantajosas, pois propiciam a produção de uma variedade de materiais que podem ser explorados pelos alunos no próprio meio ambiente.

A diversidade de estímulos disponíveis no ambiente pode despertar nos alunos um estado de motivação (SILVEIRA, 2002). Conforme Carvalho (1987), a motivação é um processo individual interno e fundamentalmente energético que determina a direção e a intensidade do aprendizado. Paralelamente à atividade do jogo associado às visitas técnicas para diagnósticos ambientais pontuais, os alunos se sentiram motivados a contar histórias e relatar casos vividos por eles, com intuito de compartilhar seus conhecimentos.

Como resultado, obteve-se uma grande aceitação do jogo por parte dos alunos e puderam-se perceber os seguintes comentários:

\footnotetext{
"Vou observar com maior atenção a lavagem das verduras, não quero pegar verminoses".

"Meu vizinho tem criação de porcos, moramos na cidade, isso não está certo, acho que vou avisar o pessoal da Vigilância Sanitária".

"[...] então é realmente importante comer apenas carnes com carimbo da inspeção".

"Nossa, não sabia que só a temperatura pode matar os cistos que estão na carne".
}

De acordo os resultados obtidos, nos questionários pré e pós-teste e nos relatórios de atividades práticas, foi constatado que os alunos aprovaram o trabalho desenvolvido e isso foi observado pelos comentários coletivos dos momentos da oficina que mais gostaram. 
Com relação às perguntas orais, feitas durante investigação na prática das oficinas sobre a construção de minhocários e terrário, foram lembrados pelos alunos os nomes populares da fauna e da flora utilizados no experimento, assim como as medidas de preservação e conservação do solo e também da água.

Houve também comentários dos alunos, os quais informaram aos amigos e familiares sobre os nomes das espécies e medidas profiláticas de preservação dos mananciais como "não jogar lixo no rio" e "não ser permitido construção de casas, viveiros, granjas e hortas, para evitar as mortes de plantas e animais". Nesse momento, observou-se a dispersão dessas informações partindo dos jovens para familiares e moradores da região, o que torna, de acordo esses resultados, evidente a justificativa deste trabalho na formação de multiplicadores de conhecimentos com relação à estrutura, importância e preservação dos recursos hídricos da região para familiares e a comunidade.

As metodologias pedagógicas que objetivam a investigação e a postura ativa dos alunos durante o processo do ensino aprendizagem são fundamentadas em dois fatores principais a serem considerados: a busca do conhecimento e a vivência do processo de ação coletiva, ou seja, a ação concreta do conhecimento obtido, através de atitudes transformadoras em seu cotidiano (PARK, 1997).

A vivência do processo de ação coletiva e colaborativa no conhecimento e transformação da realidade permite que os participantes do processo se tornem sujeitos ativos da própria história, e não sejam apenas aqueles que a contemplam e a descrevem, passam então de meros aprendizes a multiplicadores ambientais.

A proposta pedagógica de abordagem metodológica experimental deste projeto, na perspectiva construtivista trabalhada, para a realização do processo ensino-aprendizagem, pode ser entendida como alternativa viável de um ensino voltado à relação entre teoria e prática. Isso é comprovado com a observação dos resultados apresentados nas abordagens pré e pós-testes, na investigação do conhecimento dos alunos, a respeito de saneamento e recursos hídricos.

O desenvolvimento das oficinas, em seus diferentes módulos, possibilitou aos alunos a identificação de problemas ambientais do município, por meio de aulas investigativas, visitas técnicas e de diagnóstico "in loco", o qual contribuiu para a formação de multiplicadores que possam atuar de maneira crítica-cidadã junto à comunidade. 
Nesse contexto, o nosso papel como educador foi o de mediar às informações, as novas investigações e percepções em construções significativas para o processo educativo.

Apresentar e sugerir dinâmicas como as oficinas lúdico-pedagógicas conectadas à temática Educação Ambiental possibilitam alternativas quanto às atividades em sala de aula, e principalmente, enfatizam o papel do educando como sujeito/crítico/cidadão, o qual atua de forma efetiva e concreta no transformar de realidades, através da multiplicação do conhecimento adquirido.

Ao enfocar a temática ambiental, trabalhando em conjunto com as oficinas pedagógicas, na busca pela inclusão de alunos do ensino médio à iniciação científica, pela experimentação e práticas vivenciais, foi possível a construção de uma aprendizagem real, a qual pode ser concretizada nas práticas cotidianas do aluno na sociedade.

Trabalhos em Educação Ambiental são complexos, necessitam da observação dos diversos aspectos e de um resultado obtido em longo prazo. Durante o desenvolvimento deste projeto, foram observadas algumas dificuldades junto aos alunos, pois se exige tempo para integração e participação dos mesmos. Porém, todos os problemas foram superados e finalizou-se o projeto com uma esperança imensa de que trabalhos como esse sejam vistos como uma semente pequenina, cultivada em solo fértil, a qual requer cuidado, dedicação e perseverança para germinar, florescer e dar bons frutos.

\section{REFERÊNCIAS}

BARRETO, F.B.; ALMEIDA, M.J.P.M. Um diálogo com trabalho sobre experimentação nas Ciências do ensino fundamental. In: Encontro Nacional de Didática e Prática de Ensino, Goiânia, 2002.

BRASIL. Ministério da Educação e Cultura. Secretaria de Educação Fundamental. Parâmetros Curriculares Nacionais: Temas Transversais. Brasília: MEC/SEF, 1998.

CORREA, R. et. al. Educação Ambiental e Ecologia Humana. In: FONTES, L.E.F. (coord.). Metodologia em Educação Ambiental. Viçosa, 2000.

DANTAS, M. Um Projeto Ambicioso Ecologia e Desenvolvimento. Rio de Janeiro, v. 67, ano 7, p. 20-22, abr/mai, 1998.

FREIRE, P. Pedagogia do Oprimido. 17̣- ed. Rio de Janeiro: Paz e Terra, 1998. 
FROTA, P. O.; GEVERTZ, R.; DA SILVA, A. G. Como ensinar ciências. Editora Nacional. São Paulo, 1982.

JESUS JR, G. Educação Ambiental, Desenvolvimento Sustentável, Participação Popular: Breves Sugestões. In: Revista Eletrônica do Mestrado em Educação Ambiental - FURG. Vol. 2, Jul. - Ago. - Set/2000.

LAYRARGUES, P. "A resolução de problemas ambientais locais deve ser um tema gerador ou a atividade-fim da educação ambiental?" México, Siglo XXI, 1999.

LOZANO, M.S.A Educação Ambiental em uma Escola da Rede de Ensino no Município de Santo André: Análise Situacional. V. 14; jan./jun. São Paulo, 2005.

MEC/SEF.(1998). Parâmetros Curriculares Nacionais: Ciências Naturais. Brasília: Secretaria de Educação Fundamental.

PARK, P. Qué es la investigación-accion participativa - perspectivas teóricas y metodológicas. In: Maria C. Salazar (org.) La Investigación-Acción participativa inicios y desarrollos. Lima: Tarea, 1997, p. 135-174.

PHILIPPI JR, A.; MALHEIROS, T.F. Saneamento e saúde pública: integrando homem e meio ambiente. In: PHILIPPI JR, A. Saneamento saúde e ambiente: fundamentos para um desenvolvimento sustentável. Barueri, SP: Manole, 2005.

PHILIPPI Jr., A. e PELICIONI, M.C.F. Alguns pressupostos da educação ambiental. In: Philippi Jr., A. e Pelicioni, M.C.F. (editores). Educação ambiental: desenvolvimento de cursos e projetos. São Paulo: USP, Signus, 2002.

SEGURA, D.S.B. Educação Ambiental na Escola Pública: da curiosidade ingênua à consciência crítica. 1를 edição. São Paulo: Annablume/FAPESP, 2001.

SILVESTRE, M.G. Aulas Práticas de Ciências. Edunioeste: Cascavel-PR, 2001.

VIGOTSKY, L. A Formação social da mente. Editora: Guanabara. São Paulo, 1991.

ZOBILO, G. Práticas de Ensino, subsídio da atividade docente. Ática, $2^{\mathrm{a}}$ edição: São Paulo, 1994. 
even under the natural appearance of limits, they are natural references (geographic forms), don't let pass as a socio historical translation.

We could also affirm that the reality here is the expression and the translation of who live in the studied territory, study that developed by collective methodologies that aimed to break, or even attenuate, the vertical relation that characterize the science in its dichotomy, between the research object and the subject. We worked to elaborate a study in which the construction/translation of 'reality' was not only produced by the researchers (dominant perspective), but like a conjunction of perspectives, represented by the researchers together with the people that participated as subject of their own process.

Our simple experience to produce science in a participative way, in our point of view, try to dialog with the Bruno Latour (2001) observations, principally breaking the knowledge perception funded into the dualism represented in the expressions: subject/object and watcher/watched. This duality should be thought as necessary to the science rationality, because should be as corollary for the whole translation of one reality. Contrarily, our work tried to break this duality, but even doing so, could we get the whole reality translation, or the closest from what we could call of truth?

Bruno Latour (2001) comments about the use of maps in scientific researches, and how the scientist dominate the world; however this same world has to be translated into concepts and forms: yes, the scientists dominate the world - but since the world came until them in the form of bidirectional inscriptions, super posts and combined. If this happens with the science production, we perceive that the same happen with the ethno maps, since the references that constitute the drawn and the map are social representations that the Pataxó people do of their territory, there are codes, concepts and inscriptions that mean the living world and its spatiality.

However we find out that the ethno map Pataxó produced (even elaborated from the Pataxó point of view), was favored by the knowledge and instruments elaborated from other disciplines areas represented on the use do GPS and GIS. So, the georeferenced positions are well accepted because the precision that the equipments are known to have, but even in face of this aspect of precision available in the market, they don't spoken about the world, but build representations that sometime are pushed far way, other time approached. In the same way occur with the Pataxó's representation about their territory, when they draw their community, 
their representation, theydon't talk about the own world, but about the world as a stage of a theater where those representation are realized.

\section{CONCLUSIONS}

In this sense, we also observed the difference of the maps made without the participation of its actors, which consists in a representation of certain space determined by the community as important and not the whole space or the space determined for someone else. The confection of the ethno maps and the discussion of them generated reflections regarding the local reality. Were thought situations from current time, that deviate of the old scenario, as change in the cultivation system, the growth number of the families, and the amount and water quality.

The reflections about the space, in turn, facilitated the perception of vulnerability factors and of measures that are, or are not being taken to regarding them. In that sense, was possible to meditate as expected, the cultural, social, political and environmental aspects for the social technology construction.

In this context the digital technology is indispensable and the resources, limited. Still, the used resources were understood by the community that participated in the whole process. However, although it is still restricted the access to instruments (GPS) and to computer programs for mapping, the activity is viable for indigenous communities. The indigenous, like most Brazilians, have easiness using cell phones or devices like music and video players, that don't differ a lot in commands and handling, such like a standard GPS.

We noticed that is possible to promote the exercise of political power by local traditional people, to lead their cultural diversity and biodiversity conservation. It is important to point out that the community development in this process can be stimulated by the construction of autonomy and empowerment of those traditional people.

\section{Acknowledgements}

To the all Imbiruçu indigenous for the support in this study. Especially to the Cacique Romildo Pataxó and the Pataxó students Keyla Francis de Jesus da Conceição and Wakey Silva dos Santos (Junior Scientific Initiation Scholarship, UFVJM). To the Fundação de Amparo a Pesquisa do Estado de Minas Gerais (Fapemig) and to the National Council for Scientific and Technological Development (CNPq), for the scholarships. 


\section{REFERENCES}

ANGTHICHAY, ARARIBY, JASSANÃ, MANGUAHÃ, \& KANÁTYO. O povo Pataxó e suas histórias. 6.ed. São Paulo: Global, 47p., 2002.

BLACK, J. Mapas e história: construindo imagens do passado. 1.ed. Bauru, SP: EDUSC, 428p., 2005.

CAMPOS, R.C. Movimentos indígenas por educação: novos sujeitos socioculturais na história recente do Brasil. In: Reunião Anual da ANPED, n.23, Caxambu, 2000.

CARVALHO, A. $\mathbf{O}$ geoprocessamento na gestão ambiental em Terras Indígenas: uma experiência com etnomapeamento junto à Comissão Pró-Índio do Acre. Science Master Dissertation (Physical Geography). Geography Department, Faculdade de Filosofia, Letras e Ciências Humanas, USP, São Paulo, 127p., 2006.

CEDEFES. Centro de Documentação Eloy Ferreira da Silva. A luta dos índios pela terra: Contribuição a história indígena de Minas Gerais. 1.ed. Contagem, 120p., 1987.

CEDEFES. Centro de Documentação Eloy Ferreira da Silva. (2006). Pataxó: Quem é esse povo? Disponível em: $<$ http://www.cedefes.org.br/new/index.php?conteudo=materias/index\&secao=1\&tema $=53 \&$ materia $=1774>$ Acesso em: 15/06/2010.

CHAMBERS, R. The origins and practice of participatory rural appraisal. World Development, v. 22, n. 7, p. 953-969, 1994.

CHAVES, A. S.; COLLI, F. Processos educativos Revista Terra Viva: estratégias, ações, resultados e desafios na disseminação agroflorestal. Brasília: Ministério do Meio Ambiente. Série Sistematização v. 1, p. 22-33, 2006.

CORREIA, C. S. Etnozoneamento, etnomapeamento e diagnóstico etno ambiental: representações cartográficas e gestão territorial em Terras Indígenas no Estado do Acre. PhD Thesis (Antropology) - Antropology Department, UnB, Brasília, 420p., 2007.

FARIA, A. A. C. O uso do diagnóstico rural participativo em processos de desenvolvimento local: um estudo de caso. Dissertation (Master Science) Universidade Federal de Viçosa, UFV, Viçosa, 111p., 2000.

FREIRE, P. Pedagogia da autonomia - Saberes necessários à prática educativa. São Paulo: Paz e Terra, 37 ed., 148p., 1996.

HARLEY, J. B. Maps, knowledge, and power. In: Cosgrove, Denis \& Daniels, Stephen (org.). The iconography of landscape: Essays on the symbolic representation, design and use of post environments. Cambridge University Press, 1 ed., 162p, 1988. 
HERLIHY, P. H.; KNAPP, G. Maps of, by, and for the peoples of Latin America. In: Human organization. Journal of the Society for Applied Anthropology, v. 62, n. 4, p. 303-314, 2003.

IBGE. Instituto Brasileiro de Geografia e Estatística. (2007). <http://www.ibge.gov.br> LATOUR, B. A esperança de Pandorra: Ensaios sobre a realidade dos estudos científicos. Bauru, SP : EDUSC, 2001, 372p.

LAURIOLA, V. Ecologia global contra diversidade cultural? Conservação da natureza e povos indígenas no Brasil. O Monte Roraima entre Parque Nacional e a Terra Indígena Raposa-Serra do Sol. Revista Ambiente \& Sociedade, v. 5, n. 2, p. 165189, 2003.

LITTLE, P. Gestão territorial em Terras Indígenas: definição de conceitos e proposta de diretrizes. Secretaria de Estado de Meio Ambiente e Recursos Naturais - SEMA-AC, Secretaria Extraordinária dos Povos Indígenas - SEPI-AC e Agência de Cooperação Técnica Alemã (GTZ) no Brasil - GTZ, Rio Branco, Acre, 2006.

MELO, W. F., SILVA, S. S., TAVARES, R. A., FRESCHI, J. M., GAVAZZI, R. A., SILVA, J. F. M., PIYANKO, B., APIWTXA, C. A., BROWN, I. F. Aplicação de dados SRTM, sensoriamento remoto e SIG em etnomapeamento: o caso da Terra Indígena Kampa do Rio Amônia na fronteira Brasil-Acre/Peru-Ucayali. In: Simpósio Brasileiro de Sensoriamento Remoto, INPE, n.13, Florianópolis, SC, 2007. Disponível em: <http://marte.dpi.inpe.br/col/dpi.inpe.br/sbsr@80/2006/11.15.23.05/doc/59495956.pdf $>$ Acesso em 15/06/2010

ORLOVE, B. Mapping reeds and reading maps: the politics of representation in Lake Titicac. American Ethnologist, v. 18, n. 1, p. 3ᄀ38, 1991.

PATAXÓ, R. Encontro com o povo Pataxó de Carmesia. Grupo de Estudos dos Povos Indígenas de Minas Gerais. Universidade Federal dos Vales do Jequitinhonha e Mucuri (UFVJM). Campus I Diamantina, Brazil, 2008.

SCHWARTZMAN, S., ZIMMERMAN, B. Conservation alliances with indigenous peoples of the Amazon. Conservation Biology, v. 19, n. 3, p. 721-727, 2005.

SMITH, D. A. Participatory mapping of community lands and hunting yields among the bugle of Western Panama. Human Organization. Journal of the Society for Applied Anthropology, v. 62, n. 4, p. 332-343, 2003.

THE NATURE CONSERVATION. Etnomapeamento nas comunidades indígenas

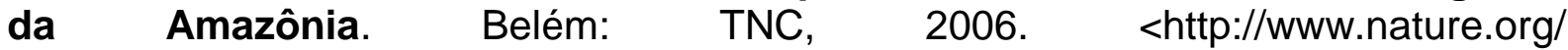
wherewework/southamerica/brasil/work/art16607.html>

VERDEJO M. E. Diagnóstico Rural Participativo. Guia Prático. 1.ed. Brasília: Ministério do Desenvolvimento Agrário, 62 p., 2006. 
VIRGILIO H, BARROS M. V. Z SIG aplicado à caracterização sócio ambiental da reserva indígena Apucarana (PR). Estudos Geográficos, v. 5, n. 1, p. 47-62, 2007. Disponível em: <http://cecemca.rc.unesp.br/ojs/index.php/estgeo>. Acesso em 15/06/2010. 